\title{
Adverse drug reactions reported by consumers for nervous system medications in Europe 2007 to 2011
}

Lise Aagaard ${ }^{1,3^{*}}$ and Ebba Holme Hansen ${ }^{2,3}$

\begin{abstract}
Background: Reporting of adverse drug reactions (ADRs) has traditionally been the sole province of healthcare professionals. In the European Union, more countries have allowed consumers to report ADRs directly to the regulatory agencies. The aim of this study was to characterize ADRs reported by European consumer for nervous system medications.
\end{abstract}

Methods: ADRs reported by consumers for nervous system medications (ATC group N) from 2007 to 2011 and located in the European ADR database, EudraVigilance, were analysed. Data were categorized with respect to age and sex, category and seriousness of reported ADRs and medications. The unit of analysis was one ADR.

Results: We located 4766 ADRs reported for nervous system medications, and one half of these were serious including 19 deaths. Less than 5\% of ADRs were reported in children. Totally, 58\% of ADRs were reported for women, $42 \%$ for men. The majority of reported ADRs were of the types "nervous system disorders" (18\% of total ADRs) followed by "psychiatric disorders" (18\% of total ADRs) and "general disorders" (15\% of total ADRs) which also were the system organ classes in which the majority of serious ADRs were found. ADR reports encompassed medicines from the therapeutic groups: antiepileptics (ATC group N03) (36\% of total ADRs), parasympathomimetics (ATC group N07) (22\% of total ADRs) and antidepressants ATC group N06A (9\% of total ADRs). Antiepileptics were the therapeutic group with the highest share of serious ADRs (60\%) followed by antidepressants (15\%). Many serious ADRs were reported for pregabalin and varenicline.

Conclusions: The majority of ADRs from nervous system mediations reported by consumers that were identified from the EudraVigilance database were serious. The value of consumer reports in pharmacovigilance still remains unclarified.

Keywords: Adverse drug reactions, Nervous system medications, Pharmacovigilance, Consumers, EudraVigilance

\section{Background}

Reporting of adverse drug reactions (ADRs) to national databases has traditionally been the sole province of health care professionals [1]. In order to strengthen the systems in some countries, consumers have also been allowed to report ADRs directly to the regulatory agencies [2]. Consumers can provide first-hand information about

\footnotetext{
* Correspondence: laagaard@health.sdu.dk

${ }^{1}$ Clinical Pharmacology, Institute of Public Health, Faculty of Health Sciences, University of Southern Denmark, J.B. Winsløws Vej 19, DK - 5000 Odense C, Denmark

${ }^{3}$ Danish Pharmacovigilance Research Project (DANPREP), Copenhagen, Denmark

Full list of author information is available at the end of the article
}

their experience with medicines and may therefore constitute a valuable information source $[1,2]$. The weakness of consumer ADR reports is the lack of medical confirmation, which might impede the interpretation of ADR causation [2]. Only few studies have analysed consumer reports submitted to ADR databases, but over the last years studies analysing ADRs reported to national pharmacovigilance databases have been published [3,4]. Medawar and Herxheimer investigated ADR reports on the risk of dependence and suicidal behaviour from paroxetine from UK consumers and healthcare professionals, respectively [5]. In 2011, McLernon et al. published a study investigating the characteristics of consumer ADRs reported in UK

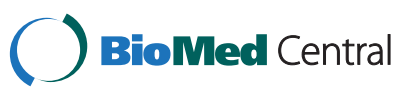


from 2008 to 2009 [6]. In Sweden, it has been possible for consumers to ADR report directly to the non-profit organization KILEN since 1978 [2], and research conducted on these data has been published in several papers and reports [7-10]. Experience with consumer reporting (2004 to 2007) in the Netherlands was recently published showing differences in the categories of seriousness and outcome of the reported ADRs between patients and healthcare professionals [11]. A study from Denmark analysing differences in ADR reporting patterns between consumers and healthcare professionals (2004 to 2006) showed that patients were more likely to report ADRs from nervous and psychiatric medications, that patients' share of reports on serious ADRs was comparable to that of physicians, and that patients provided new and unknown information about ADRs [12]. Analysis of consumer reports of suspected ADRs submitted voluntarily to the website of a Danish consumer magazine showed that consumers reported ADRs for nervous systems medications and that patients report rather unspecific symptoms, as they use lay terms to describe reactions [13]. Patients also reported several ADRs, which prescribers may not consider serious but may be troublesome to patients and therefore patients find worthy of reporting [13]. The published consumer studies which all were conducted on national datasets showed that consumers are willing to report many ADRs for nervous system medications, but we do not know to which extent the above findings are generaliserable to populations in other countries. Since 2012, researchers were allowed access to ADR data in the EU ADR database, EudraVigilance (EV) and this has opened for cross-national analysis based on a standardised reporting format [14]. The objective of this study was to investigate ADR reports submitted by consumers for nervous system medications in Europe during the first 5 years of electronic reporting to the EV ADR database.

\section{Methods \\ Setting}

EudraVigilance (EV) is the central database of reports of suspected spontaneous ADR reports and ADRs reported in clinical trials for all medicinal products authorized in the European Economic Area (EEA) [15]. In compliance with the EU pharmacovigilance legislation, ADRs are reported to EV by regulatory agencies in member states where the ADR occurred. EV was set up in December 2001 to facilitate the electronic reporting of ADRs in the EEA. Data should be transmitted in accordance with the ICH E2B (R2) standard [15]. The minimum information required for an $\mathrm{ADR}$ report to enter the EV database is the following parameters: type of reporter, patient, at least one suspected active substance/medicinal product, and at least one suspected ADR (Volume 9A) [15]. The EV database is not publically accessible, and authorisation for data access was given by the European Medicines Agency. By 2012, consumer reporting was officially accepted in 5 European countries: Denmark, the Netherlands, Norway, Sweden and the United Kingdom [1]. Before July 2012, countries were only requested to forward serious consumer ADR reports to the EV database [16].

\section{Study design}

The study comprised all ADR reports occurring from 2007 to 2011, located in the EV database and reported by consumers for nervous system medications (ATC group N). The content of the reports was analysed with respect to seriousness, categories of ADRs classified by system organ class (SOC) and medications. The unit of analysis was one ADR. Patients' age was dichotomized into two groups: children (0-17-year-olds) and adults $(18+)$.

\section{Material}

ADR information was provided for this study in anonymous form with encrypted identification [8]. Data extraction and data analyses of the raw material were comprehensive and time-consuming. Information was extracted from the ADR database on the date reports were received; category of persons submitting the reports; and criteria of seriousness and medications for which the ADRs were reported. The reported ADRs were coded according to type and seriousness using CIOMS (Council for International Organizations of Medical Sciences) criteria by academic staff in the national regulatory agencies [16]. ADR data was placed at the disposal of this study in anonymous form with encrypted identification of the medicine user. Data were extracted from the EV database in Microsoft Excel files using the following criteria: patient's sex and age, medicines (active substance), adverse drug reaction and severity. EMA has to ensure that, in complying with regulation (EC) $1049 / 2001$, the protection of privacy and integrity of individuals is guaranteed, and therefore individual country specific ADR information was not disclosed [17]. The material comprised all ADRs reports from consumers reported to the EV database from 2007 to 2011. Data were extracted from the EV database and delivered to us as several large Excel files. Data comprised all ADR reports form consumers located in the EV database by 14 March 2012. In STATA $^{\oplus}$ (statistical software package) the Excel files were merged into one major file and the ADR reports were searched for duplicates. Data analysis including coding of ADR reports was conducted in an Access database. Each ADR report may refer to one or more suspected ADR (s) as well as to one or more medicinal products. In this study we included ADRs reported for medications, which were listed as suspect drug by the reporter, meaning that the reporter suspected this drug and not the concomitant medicine to have caused the ADR. 
Table 1 Fatal consumer cases reported for nervous systems medications in Europe, 2007 to 2011

\begin{tabular}{|c|c|c|c|c|c|}
\hline Case no. & Medicine (s) & ATC group & Adverse drug reaction (s) & Sex (M/F) & Age \\
\hline 1 & Diamorphine & N02AA09 & Sudden death & $\mathrm{F}$ & $18+$ \\
\hline \multirow[t]{4}{*}{2} & Metamizole & $\mathrm{N} 02 \mathrm{BB} 02$ & Agranulocytosis & $\mathrm{F}$ & $18+$ \\
\hline & & & Leukopenia & & \\
\hline & & & Multi-organ failure & & \\
\hline & & & Sepsis/septic shock & & \\
\hline 3 & Morphine & N02AA01 & Cerebrovascular accident & $\mathrm{F}$ & $18+$ \\
\hline 4 & Oxycodone & N02AA05 & Intentional overdose/suicidal ideation & M & $18+$ \\
\hline 5 & Apomorphine & N04BC07 & Pneumonia & M & NA \\
\hline \multirow[t]{2}{*}{6} & Apomorphine & N04BC07 & Intestinal haemorrhage & M & NA \\
\hline & & & Pneumonia aspiration & & \\
\hline \multirow[t]{3}{*}{7} & Apomorphine & N04BC07 & Anaemia & $\mathrm{F}$ & $18+$ \\
\hline & & & Haematocrit decreased & & \\
\hline & & & Red blood cell sedimentation rate increased & & \\
\hline 8 & Apomorphine & N04BC07 & Death & $\mathrm{F}$ & NA \\
\hline 9 & Apomorphine & N04BC07 & Death & $\mathrm{F}$ & $18+$ \\
\hline 10 & Apomorphine & N04BC07 & Death & $\mathrm{F}$ & NA \\
\hline 11 & Apomorphine & N04BC07 & Death & $\mathrm{F}$ & $18+$ \\
\hline \multirow[t]{2}{*}{12} & Carbidopa/levodopa & N04BA02 & Death & M & $18+$ \\
\hline & Entacapone, Rotigotine & & & & \\
\hline \multirow[t]{4}{*}{13} & Clomethiazole & N05CM02 & Leucocytosis & $\mathrm{F}$ & NA \\
\hline & & & Pyrexia & & \\
\hline & & & Musculoskeletal stiffness & & \\
\hline & & & Neuroleptic malignant syndrome & & \\
\hline \multirow[t]{2}{*}{14} & Clozapine & N05AH02 & Cardiac failure & $\mathrm{F}$ & $18+$ \\
\hline & & & Somnolence & & \\
\hline 15 & Citalopram & N06AB04 & Fatigue/malaise & $\mathrm{F}$ & NA \\
\hline 16 & Duloxetine & N06AX21 & Deafness & $\mathrm{F}$ & $18+$ \\
\hline
\end{tabular}

Abasia

Urinary tract infection

Septic shock

Urosepsis

Hyponatraemia

Neoplasm malignant

Aphasia

Urinary incontinence

Renal failure

Depressed level of consciousness/sedation

Tachyphrenia

Completed suicide

Dependence

Indifference 
Table 1 Fatal consumer cases reported for nervous systems medications in Europe, 2007 to 2011 (Continued)

\begin{tabular}{|c|c|c|c|c|c|}
\hline 18 & Amitriptyline & N06AA09 & Toxicity to various agents & M & NA \\
\hline \multirow[t]{2}{*}{19} & Rivastigmine & N06DA03 & Lung infection & $\mathrm{F}$ & $18+$ \\
\hline & & & Mood altered/aggression & & \\
\hline
\end{tabular}

M: male, F: female, NA:no information available.

\section{Classification of ADRs by type}

The different types of reported ADRs were classified according to the Medical Dictionary for Regulatory Activities (MedDRA) System Organ Class (SOC) [18]. Serious ADRs were defined as: fatal, life-threatening, requiring hospitalisation or prolongation of existing hospitalisation, resulting in persistent or significant disability/incapacity in the reporter's assessment, in a congenital anomaly/birth defect and other medically important conditions. All other ADRs are classified as non-serious [18].

\section{Classification of medications by anatomical therapeutic chemical (ATC) group}

The ATC system is a system for classifying medicinal products according to their primary constituent, the organ or system on which they act and their chemical, pharmacological and therapeutic properties [19]. Medicinal products are classified at five different levels. The medicines are divided into 14 main groups (first level), with one pharmacological/therapeutic subgroup (second level), and the fifth level is the chemical substance [18]. As the ADR data provided by EMA did not contain any information about ATC codes, these were added manually to the data file. The medicinal products reported are referenced based on their active substance and in this article we present ADR data at ATC level 1 and 5 [19].

\section{Results}

From 2007 to 2011, a total of 7434 consumer ADR reports containing information about 35349 ADRs was located in $\mathrm{EV}$. Of these, 4766 ADRs were submitted for nervous system medications. In total, $51 \%$ of ADRs were classified as serious and of these 19 fatal cases were reported. The characteristics of the fatal cases are displayed in Table 1. The largest number of fatal cases $(n=8)$ was reported for apomorphine (ATC group N04) followed by five fatal cases reported for antidepressants (ATC group N06). Totally, $58 \%$ of ADRs were reported for women and $42 \%$ for men. Less than $5 \%$ of ADRs were reported in children.

\section{ADRs by type and seriousness}

Table 2 shows the distribution of reported ADRs by SOC. In total, consumers reported 26 ADR categories. The largest shares of ADRs were reported for the SOCs: nervous system disorders (18\% of total ADRs), psychiatric disorders (18\% of total ADRs); and general disorders and administration site conditions (15\% of total ADRs).
The largest share of serious ADRs was of the type psychiatric disorders (23\% of serious) followed by nervous system disorders (17\% of serious) and ADRs of the general type (12\% of ADRs).

\section{ADRs by therapeutic groups}

Table 3 displays the number of ADRs reported by consumers distributed on therapeutic groups and seriousness. Reports encompassed medicines from the therapeutic groups: antiepileptics (ATC group N03) (36\%),

Table 2 Number of consumer adverse drug reactions for nervous system medications in Europe by type and seriousness, 2007 to 2011

\begin{tabular}{ll}
\hline System organ class (descending order) & Number (serious) \\
\hline Psychiatric disorders & $868(547)$ \\
Nervous system disorders & $847(424)$ \\
General disorders and administration site conditions & $736(303)$ \\
Gastrointestinal disorders & $651(199)$ \\
Investigations & $251(151)$ \\
Skin and subcutaneous tissue disorders & $236(118)$ \\
Musculoskeletal and connective tissue disorders & $219(96)$ \\
Injury, poisoning and procedural complications & $145(93)$ \\
Eye disorders & $142(65)$ \\
Respiratory, thoracic and mediastinal disorders & $123(69)$ \\
Cardiac disorders & $79(57)$ \\
Vascular disorders & $74(58)$ \\
Metabolism and nutrition disorders & $69(44)$ \\
Renal and urinary disorders & $60(38)$ \\
Ear and labyrinth disorder & $56(29)$ \\
Infections and infestations & $50(29)$ \\
Reproductive system and breast disorders & $44(15)$ \\
Blood and lymphatic system disorders & $21(21)$ \\
Social circumstances & $19(14)$ \\
Surgical and medical procedures & $18(17)$ \\
Hepatobiliary disorders & $17(15)$ \\
Immune system disorders & $16(10)$ \\
Neoplasm benign, malignant and unspecified & $10(10)$ \\
Endocrine disorders & $9(9)$ \\
\hline
\end{tabular}


Table 3 Consumer adverse drug reactions ( $N$ ) for nervous system medications in Europe by therapeutic group and seriousness (in parentheses), 2007 to 2011

\begin{tabular}{|c|c|c|}
\hline Therapeutic group (ATC level 2) & Substance & Total (serious) \\
\hline \multirow[t]{6}{*}{ Anaesthetics (N01) } & Articaine & $1(1)$ \\
\hline & Bupivacaine & $4(4)$ \\
\hline & Fentanyl & $24(24)$ \\
\hline & Propofol & $2(2)$ \\
\hline & Sevoflurane & $2(2)$ \\
\hline & Sufentanil & $4(4)$ \\
\hline Total N01 & & $37(37)$ \\
\hline \multirow[t]{20}{*}{ Analgesics (N02) } & Buprenorphine & $24(24)$ \\
\hline & Codeine & $14(14)$ \\
\hline & Diamorphine & $3(3)$ \\
\hline & Dihydroergotamine & $27(27)$ \\
\hline & Ergotamine & $2(2)$ \\
\hline & Flupirtine & $6(6)$ \\
\hline & Frovatriptan & $2(2)$ \\
\hline & Hydromorphone & $22(22)$ \\
\hline & Metamizole & $20(20)$ \\
\hline & Methylergometrine & $2(2)$ \\
\hline & Methysergide & $25(25)$ \\
\hline & Morphine & $6(6)$ \\
\hline & Oxycodone & $56(56)$ \\
\hline & Paracetamol & $42(42)$ \\
\hline & Phenazone & $8(8)$ \\
\hline & Pizotifen & $2(2)$ \\
\hline & Propyphenazone & $7(7)$ \\
\hline & Sumatriptan & $3(3)$ \\
\hline & Tilidine & $11(10)$ \\
\hline & Tramadol & $62(57)$ \\
\hline Total N02 & & $344(338)$ \\
\hline \multirow[t]{12}{*}{ Antiepileptic drugs (N03) } & Carbamazepine & 142(142) \\
\hline & Clonazepam & $13(13)$ \\
\hline & Gabapentin & $53(53)$ \\
\hline & Lamotrigine & $78(78)$ \\
\hline & Levetiracetam & $2(2)$ \\
\hline & Oxcarbazepine & $36(36)$ \\
\hline & Phenytoin & $6(6)$ \\
\hline & Phenobarbital & $4(4)$ \\
\hline & Pregabalin & 1510(1510) \\
\hline & Topiramate & $6(6)$ \\
\hline & Valproate & $17(17)$ \\
\hline & Zonisamide & $3(3)$ \\
\hline Total N03 & & 1870(1870) \\
\hline Antiparkinson drugs (N04) & Amantadine & 9(9) \\
\hline
\end{tabular}

Table 3 Consumer adverse drug reactions $(\mathrm{N})$ for nervous system medications in Europe by therapeutic group and seriousness (in parentheses), 2007 to 2011 (Continued)

\begin{tabular}{|c|c|c|}
\hline & Apomorphine & $45(45)$ \\
\hline & Benserazide & $1(1)$ \\
\hline & Bromocriptine & $26(26)$ \\
\hline & Cabergoline & $1(1)$ \\
\hline & Carbidopa & $43(43)$ \\
\hline & Entacapone & 39(39) \\
\hline & Levodopa & $44(44)$ \\
\hline & Piribedil & $1(1)$ \\
\hline & Pramipexole & $17(17)$ \\
\hline & Procyclidine & $7(7)$ \\
\hline & Rasagiline & $15(15)$ \\
\hline & Rotigotine & $1(1)$ \\
\hline & Ropinirole & $15(15)$ \\
\hline Total N04 & & $264(264)$ \\
\hline Antipsychotics (N05A) & Amisulpride & $1(1)$ \\
\hline & Aripiprazole & 19(19) \\
\hline & Bromperidol & $8(8)$ \\
\hline & Chlorpromazine & $7(7)$ \\
\hline & Chlorprothixene & 23(23) \\
\hline & Clozapine & $71(71)$ \\
\hline & Flupentixol & $1(1)$ \\
\hline & Fluspirilene & $2(2)$ \\
\hline & Haloperidol & $12(12)$ \\
\hline & Levomepromazine & $9(9)$ \\
\hline & Lithium & 18(18) \\
\hline & Melperone & $7(7)$ \\
\hline & Olanzapine & 23(23) \\
\hline & Perphenazine & $12(12)$ \\
\hline & Pipamperone & $3(3)$ \\
\hline & Quetiapine & $46(46)$ \\
\hline & Risperidone & $37(37)$ \\
\hline & Sulpiride & $7(7)$ \\
\hline & Thioridazine & $6(6)$ \\
\hline & Tiapride & $6(6)$ \\
\hline & Zuclopenthixol & $21(21)$ \\
\hline Total N05A & & 339(339) \\
\hline Anxiolytics (N05B) & Alprazolam & $17(17)$ \\
\hline & Bromazepam & $14(14)$ \\
\hline & Chlordiazepoxide & $2(2)$ \\
\hline & Clorazepate & $9(9)$ \\
\hline & Diazepam & 19(19) \\
\hline & Lorazepam & $74(74)$ \\
\hline & Oxazepam & $8(8)$ \\
\hline
\end{tabular}


Table 3 Consumer adverse drug reactions $(\mathrm{N})$ for nervous system medications in Europe by therapeutic group and seriousness (in parentheses), 2007 to 2011 (Continued)

\begin{tabular}{|c|c|c|}
\hline Total N05B & & 143(143) \\
\hline \multirow[t]{7}{*}{ Hypnotics and sedatives (N05C) } & Butalbital & $5(5)$ \\
\hline & Clomethiazole & $5(5)$ \\
\hline & Flunitrazepam & $1(1)$ \\
\hline & Melatonin & $4(4)$ \\
\hline & Zaleplon & $3(3)$ \\
\hline & Zolpidem & $14(14)$ \\
\hline & Zopiclone & $12(12)$ \\
\hline Total N05C & & $44(44)$ \\
\hline \multirow[t]{17}{*}{ Antidepressants (N06A) } & Agomelatine & $29(29)$ \\
\hline & Amitriptyline & $11(11)$ \\
\hline & Bupropion & $7(7)$ \\
\hline & Citalopram & $38(38)$ \\
\hline & Clomipramine & $5(5)$ \\
\hline & Duloxetine & $27(27)$ \\
\hline & Doxepin & $1(1)$ \\
\hline & Escitalopram & $25(25)$ \\
\hline & Fluoxetine & $14(14)$ \\
\hline & Imipramine & $1(1)$ \\
\hline & Mirtazapine & $18(18)$ \\
\hline & Nortriptyline & $6(6)$ \\
\hline & Opipramol & $28(28)$ \\
\hline & Paroxetine & $21(21)$ \\
\hline & Sertraline & $22(22)$ \\
\hline & Trimipramine & $7(7)$ \\
\hline & Venlafaxine & $217(177)$ \\
\hline Total N06A & & $477(437)$ \\
\hline \multirow[t]{2}{*}{ Psychostimulants (N06B) } & Caffeine & $16(16)$ \\
\hline & Methylphenidate & $61(61)$ \\
\hline Total N06B & & $77(77)$ \\
\hline \multirow[t]{2}{*}{ Anti-dementia drugs (N06D) } & Memantine & 2(2) \\
\hline & Rivastigmine & $50(50)$ \\
\hline Total N06D & & $52(52)$ \\
\hline \multirow[t]{4}{*}{ Parasympathomimetics (N07) } & Disulfiram & $11(11)$ \\
\hline & Methylnaltrexone & 2(2) \\
\hline & Nicotine & $91(91)$ \\
\hline & Varenicline & 1017(135) \\
\hline Total N07 & & $1121(239)$ \\
\hline Total ATC group N & & $4766(2433)$ \\
\hline
\end{tabular}

parasympathomimetics (ATC group N07) (22\%) and antidepressants ATC group N06A (9\%). Except from parasympathomimetics, the majority of ADRs were serious. In particular, a large number of ADRs were reported for prebagalin $(\mathrm{n}=1510)$ and varenicline $(\mathrm{n}=1017)$. The most commonly reported ADRs for venlafaxine were anxiety, restlessness, paraesthesia and sleep disorder. Table 4 displays characteristics of serious ADRs reported for pregabaline. In total, $50 \mathrm{ADR}$ categories were reported; the most frequently reported ADRs were drug ineffective/drug effect decreased $(n=83)$, dizziness $(n=78)$, pain $(n=61)$, somnolence $(n=56)$ and fatigue $(n=54)$. Table 5 displays the characteristics of serious ADRs reported for varenicline. The largest number of reported ADRs was musculoskeletal pain $(n=9)$, sleep disorder $(n=7)$, chest disorder/pain $(n=7)$, depression $(n=6)$ and suicidal behavior/ideation $(n=6)$.

\section{Discussion}

This is the first study to systematically analyse ADRs for nervous system medications reported by consumers to the EV database. Almost all ADRs, except for those reported for parasympathomimetics, were serious and several fatal cases were reported. Reported ADRs were predominantly of the type nervous and psychiatric disorders and general disorders. The majority of ADRs were reported for pregabalin, varenicline and venlafaxine.

\section{ADRs by type and seriousness}

The most frequently reported ADRs for nervous system medications were of the type nervous and psychiatric disorders and this finding was expected due to the mechanism of action of the reported nervous system medications. Additionally, a large number of ADRs of the type general disorders and administration site conditions and gastrointestinal disorders were reported, and this finding was also in line with results in previous consumer studies [5-13]. More than one half of reported ADRs were serious, however this reporting pattern was not surprising, since countries were not requested to report non-serious ADRs to the EV database during the study period [16].

\section{ADRs by therapeutic groups}

The largest number of ADRs was reported for antiepileptics and antidepressants, which can be explained by the frequent use of these medications in adults [20]. A high number of ADRs were reported for varenicline but only few were serious. In 2007, based on consumer reports in the USA, there was a high media attention on the increased risk of serious ADRs such as suicidal ideation and occasional suicidal behaviour, erratic behaviour and drowsiness reported for varenicline leading to black box warnings in the USA (July 2009) [21]. The ADR signal was later confirmed in a meta-analysis [22]. The high number of ADRs reported for varenicline by European consumers could have been stimulated by this media attention; however, the majority of reported ADRs were 
Table 4 Serious adverse drug reactions reported for pregabalin by European consumers, 2007 to 2011

Adverse drug reaction(s)

Drug ineffective/drug effect decreased

Dizziness

Pain

Somnolence

Fatigue

Weight changes

Abdominal pain

Nausea

Headache

Vision blurred

Insomnia

Muscle spasms

Oedema

Gait disturbance

Myalgia

Hyperhidrosis

Appetite changes

Dry mouth

Malaise

Pruritus

Constipation

Disturbance in attention

Depression

Rash

Balance disorder

Memory impairment

Paraesthesia

Vertigo

Withdrawal syndrome

Accidental exposure

Diarrhoea

Feeling abnormal

Speech disorder

Anxiety

Arthralgia

Feeling drunk

Tremor

Eye swelling

Nasal congestion

Burning sensation

Erectile dysfunction

Urinary tract disorder
Table 4 Serious adverse drug reactions reported for pregabalin by European consumers, 2007 to 2011 (Continued)

\begin{tabular}{ll}
\hline Vomiting & 10 \\
Others $(n<10)$ & 492 \\
Total & 1510 \\
\hline
\end{tabular}

non-serious. For pregabalin a large number of the ADRs "drug ineffective/drug effect decreased" were reported, probably because this side effect can easily be assessed, and is very obvious compared to many other types of ADRs. To evaluate whether ADRs reported for pregabalin and varenicline can act as early warning for new ADR signals more in-depth analysis of the ADR reports should be conducted.

Strengths and limitations of this study

The strength of this study is that data comprised all ADRs reported by consumers in Europe, which were

Table 5 Serious adverse drug reactions reported for varenicline by European consumers, 2007 to 2011

\begin{tabular}{ll}
\hline Adverse drug reaction(s) & $\mathbf{N}$ \\
\hline Musculoskeletal pain & 9 \\
Sleep disorder & 7 \\
Chest discomfort/pain & 7 \\
Depression & 6 \\
Suicidal behaviour/ideation & 6 \\
Nausea & 5 \\
Rash & 4 \\
Aggression & 4 \\
Mood altered/mood swings & 3 \\
Feeling abnormal & 3 \\
Headache & 3 \\
Oropharyngeal blistering/pain & 3 \\
Anxiety & 3 \\
Hallucination & 3 \\
Tearfulness & 2 \\
Fatigue & 2 \\
Pyrexia & 2 \\
Epilepsy & 2 \\
Movement disorder & 2 \\
Muscle spasms/weakness & 2 \\
Abdominal discomfort/pain & 2 \\
Erythema & 2 \\
Othersersensitivity $(n<2)$ & 2 \\
Total & 2 \\
\hline & 2 \\
\hline & 2 \\
\hline & 2 \\
\hline
\end{tabular}


forwarded to the EV database during a five-year period and present in the database by March 2012. A major limitation to this study is that we do not know to which extent the causality of these ADRs can be confirmed, and this has implications for the interpretation of the findings [2]. The value of consumer reports in detection of new ADR signals remains unclarified due to the lack of information about causality. In this study, we did not evaluate the validity of the consumer reports since we only had access to the data entered into the EV database and not the original reports. Spontaneous reporting systems suffer from various barriers, such as incomplete recognition of ADRs, administrative barriers to reporting and low data quality, all of which may result in underreporting of important serious and rare events [2]. ADRs that are non-serious or already known may be overreported; however, this study provides information on reported ADRs, and this information contributes to broadening the knowledge on medicine safety. Before July 2012 countries were only obliged to report serious consumer reports to $\mathrm{EV}$, which may explain the large number of serious ADRs found, and the low number of non-serious consumer reports. Therefore there may be additional non-serious consumer ADR reports present in the regulatory agencies. With the new pharmacovigilance regulation that came into force in July 2012 the share of serious consumer reports in EV will probably decline although the total number of consumer reports is expected to increase.

Hence, it is not possible to generalize from data reported to the EV database to the other EU member states. Spontaneous reports are an important source of information about new and previously unrecognized ADRs, and the value of spontaneous reporting schemes lies in their ability to act as hypothesis-generating procedures [2]. Therefore, EMA should continue to systematically survey and analyse ADRs reported by consumers in order to signal previously unknown ADRs. Another important issue to be investigated in future studies is to which extent individuals suffering from ADRs later recover from the reported reactions.

\section{Conclusion}

The majority of ADRs from nervous system mediations reported by consumers that were identified from the EudraVigilance database were serious. The value of consumer reports in pharmacovigilance still remains unclarified.

\section{Competing interests}

The authors have not received reimbursements, fees, funding, or salary from an organization that may in any way gain or lose financially from the publication of this manuscript, either now or in the future. The authors do not hold stocks or shares in an organization that may in any way gain or lose financially from the publication of this manuscript. The authors do not hold or plan to apply for any patents relating to the content of the manuscript. The authors have not received reimbursements, fees, funding, or salary from an organization that holds or has applied for patents relating to the content of the manuscript. The authors declare no other non-financial competing interests.

\section{Authors' contribution}

$\mathrm{LA}$ and $\mathrm{EHH}$ designed the study, analysed data and wrote the first version of the manuscript. LA carried out the sampling. Both authors saw and approved the final version of the manuscript. No sources of funding were used to assist in the preparation of this study.

\section{Acknowledgements}

The authors would like to thank the European Medicines Agency for providing data and MSc Jesper Frederiksen for assistance with data handling.

\section{Author details}

${ }^{1}$ Clinical Pharmacology, Institute of Public Health, Faculty of Health Sciences, University of Southern Denmark, J.B. Winsløws Vej 19, DK - 5000 Odense C, Denmark. ${ }^{2}$ Section for Social and Clinical Pharmacy, Department of Pharmacy, Faculty of Health and Medical Sciences, University of Copenhagen, Copenhagen, Denmark. ${ }^{3}$ Danish Pharmacovigilance Research Project (DANPREP), Copenhagen, Denmark.

Received: 18 October 2012 Accepted: 11 June 2013

Published: 13 June 2013

\section{References}

1. van Hunsel F, Härmark L, Pal S, Olsson S, van Grootheest K: Experiences with adverse drug reaction reporting by patients: an 11-country survey. van Hunsel F, Härmark L, Pal S, Olsson S, van Grootheest K. Drug Saf 2012, 35:45-60.

2. Blenkinsopp A, Wilkie P, Wang M, Routledge PA: Patient reporting of suspected adverse drug reactions: a review of published literature and international experience. Br J Clin Pharmacol 2006, 63:148-156.

3. Hawcutt DB, Mainie P, Riordan A, Smyth RL, Pirmohamed M: Reported paediatric adverse drug reactions in the UK 2000-2009. Br J Clin Pharmacol 2012, 73:437-446.

4. Durrieu G, Palmaro A, Pourcel L, Caillet C, Faucher A, Jacquet A, Ouaret S, Perault-Pochat MC, Kreft-Jais C, Castot A, Lapeyre-Mestre M, Montastruc J: First French experience of ADR reporting by patients after a mass immunization campaign with Influenza A (H1N1) pandemic vaccines: a comparison of reports submitted by patients and healthcare professionals. Drug Saf 2012, 35:845-854.

5. Medawar $\mathrm{C}$, Herxheimer $\mathrm{A}$ : A comparison of adverse drug reactions from professionals and users, relating to risk of dependence and suicidal behaviour with paroxetine. Int J Risk Saf Med 2004, 16:5-19.

6. McLernon DJ, Bond CM, Hannaford PC, Watson MC, Lee AJ, Hazell L, Avery A: Adverse drug reaction reporting in the UK: a retrospective observational comparison of yellow card reports submitted by patients and healthcare professionals. Drug Saf 2010, 33:775-788.

7. KILEN: Consumer reports on medicines (CRM) - consensus document. ; 2000. Available at http:/www.kilen.org/indexe.htm (last accessed 21 March 2013).

8. Health Action International Europe: Patient reporting of adverse drug reactions - seminar report; 2005. Available at http://www.haiweb.org/ docs2005/final_report.doc (last accessed 21 March 2013).

9. Vilhelmsson A, Svensson T, Meeuwisse A, Carlsten A: What can we learn from consumer reports on psychiatric adverse drug reactions with antidepressant medication? Experiences from reports to a consumer association. BMC Clin Pharmacol 2011, 25:11. doi:10.1186/1472-6904-11-16. 16.

10. Vilhelmsson A, Svensson T, Meeuwisse A, Carlsten A: Experiences from consumer reports on psychiatric adverse drug reactions with antidepressant medication: a qualitative study of reports to a consumer association. BMC Pharmacol Toxicol. 2012, 13:19. doi:10.1186/2050-6511-13-19.

11. De Langen J, Van Hunsel F, Passier A, de Jong-van den Berg L, van Groothest K: Adverse drug reaction reporting by patients in the Netherlands. Three years of experience. Drug Saf 2008, 31:515-554.

12. Aagaard L, Nielsen LH, Hansen EH: Consumer reporting of adverse drug reactions. A retrospective analysis of Danish adverse drug reaction database from 2004 to 2006. Drug Saf 2009, 32:1067-1074. 
13. Aagaard L, Hansen EH: Consumers' reports of suspected adverse drug reactions volunteered to a consumer magazine. Br J Clin Pharmacol 2010, 69:317-318.

14. EU directive of the European Parliament and of the council amending Directive 2001/83/EC as regards information to the general public on medicinal products subject to medical prescription. COM (2012) 48 final. Brussels. 10.2.2012. Available at: http://eur-lex.europa.eu/index.htm (last accessed 21 March 2013).

15. European Medicines Agency: Note for quidance - EudraVigilance Human Processing of safety messages and individual case safety reports (ICSRs). EMA H/20665/04/Final Rev. 2. Available at: http://eudravigilance.ema.europa.eu/ human/euPoliciesAndDocs03.asp (last accessed 21 March 2013).

16. Volume 9. Pharmacovigilance: medicinal products for human use and veterinary products. Available at http://ec.europa.eu/enterprise/ pharmaceuticals/eudralex/homev9.htm (last accessed 3 April 2012).

17. Office Journal of the European Commission: Regulation (EC) No 1049/2001 of the European Parliament and of the Council of 30 May 2001 regarding public access to European Parliament, Council and Commission. L 145/43. Available at: www.europarl.europa.eu/register/pdf/r1049_en.pdf (last accessed 21 March 2013)

18. MedDRA. Available at http://www.meddramsso.com (last accessed 5 May 2012).

19. WHO collaboration centre for drug statistics methodology. 2007. Available at http://www.whocc.no/atc_ddd_index/ (last accessed 3 April 2012).

20. Danish national registry of medicinal products statistics. http://www. medstat.dk/ (last accessed 3 April 2012).

21. FDA: FDA requires new box warnings for the smoking cessation drugs Chantix and Zyban. Available at: http://www.fda.gov/Drugs/DrugSafety/ DrugSafetyPodcasts/ucm170906.htm (last accessed 21 March 2013).

22. Singh S, Loke YK, Spangler JG, Furberg CD: Risk of serious adverse cardiovascular events associated with varenicline: a systematic review and meta-analysis. CMAJ 2011, 83:1359-1366.

doi:10.1186/2050-6511-14-30

Cite this article as: Aagaard and Hansen: Adverse drug reactions reported by consumers for nervous system medications in Europe 2007 to 2011. BMC Pharmacology and Toxicology 2013 14:30.

\section{Submit your next manuscript to BioMed Central and take full advantage of:}

- Convenient online submission

- Thorough peer review

- No space constraints or color figure charges

- Immediate publication on acceptance

- Inclusion in PubMed, CAS, Scopus and Google Scholar

- Research which is freely available for redistribution 